\title{
Electrooptical Modulator Fabricated by Gallium Diffusion in Lithium Niobate
}

\author{
Wen-Hung Huang, Student Member, IEEE, Chia-Wei Lin, Student Member, IEEE, and \\ Way-Seen Wang, Member, IEEE
}

\begin{abstract}
Mach-Zehnder modulators fabricated by gallium diffusion in $y$-cut lithium niobate $\left(\mathrm{LiNbO}_{3}\right)$ are presented. The measured halfwave voltages are 6.2 and $3.2 \mathrm{~V}$ when the electrode lengths are 0.4 and $0.8 \mathrm{~cm}$, respectively. By calculating the overlap integral between the modulating electric and the guided optical fields, the value of the electrooptic coefficient $r_{33}$ is found in good agreement with that of the bulk $\mathrm{LiNbO}_{3}$, which indicates no significant degradation in $r_{33}$ is induced by the in-diffused gallium atoms.
\end{abstract}

Index Terms-Electrooptic coefficient, gallium-diffused waveguide, lithium niobate, Mach-Zehnder modulator.

\section{INTRODUCTION}

$\mathbf{E}$ LECTROOPTICAL (EO) device in lithium niobate $\left(\mathrm{LiNbO}_{3}\right)$ substrate, known for its low optical loss and driving voltage, is one of the key components in the optical communication system. Moreover, with high modulation bandwidth and good material stability, optical modulators based on $\mathrm{LiNbO}_{3}$ waveguides have been widely used in high-speed optical networks. With an anisotropic electrooptic tensor, $\mathrm{LiNbO}_{3}$ optical modulators are polarization sensitive so that the polarization of the input waves must be carefully controlled.

As the widely used titanium-diffused $\mathrm{LiNbO}_{3}$ waveguides support both polarization states, good control of the polarizations is an important issue. To fabricate a polarization-insensitive optical modulator in $\mathrm{LiNbO}_{3}$ substrate, there are two general solutions. The first one [1], [2] is independent control of the modulation for each polarization, while a complex design of electrode configuration is needed. The other one [3], [4] is to fabricate the waveguide with a propagation direction along the optical axis. However, the largest EO coefficient $r_{33}$ is not used and thus, the driving voltage is higher.

It is known that waveguides supporting only ordinary or extraordinary waves are good alternatives. Conventionally, proton-exchange waveguides supporting only extraordinary waves are used. However, the EO coefficients are significantly reduced after the proton exchange process [5] such that an ad-

Manuscript received January 4, 2008; revised April 9, 2008. This work was supported by the National Science Council, Taipei, Taiwan, R.O.C., under Contract NSC 96-2221-E-002-096.

W.-H. Huang is with the Graduate Institute of Photonics and Optoelectronics, National Taiwan University, Taipei 106, Taiwan, R.O.C.

C.-W. Lin is with the Graduate Institute of Electronics Engineering, National Taiwan University, Taipei 106, Taiwan, R.O.C.

W.-S. Wang is with the Department of Electrical Engineering, National Taiwan University, Taipei 106, Taiwan, R.O.C. (e-mail: wswang@cc.ee.ntu. edu.tw).

Digital Object Identifier 10.1109/LPT.2008.924902

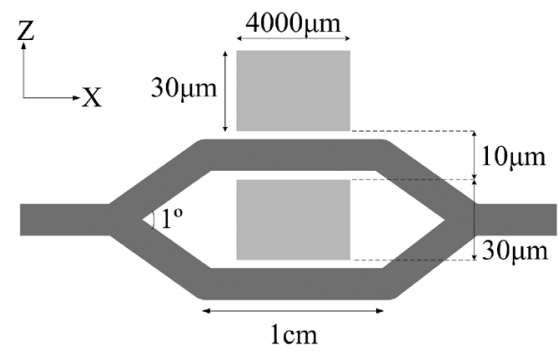

Fig. 1. Schematic diagram of the proposed gallium-diffused Mach-Zehnder interferometer.

ditional thermal annealing process is needed [6]. However, the annealing process is time-consuming and needs to be carefully controlled. Recently, gallium-diffused waveguides supporting only the extraordinary wave have been proposed and are good alternatives for singly polarized waveguides [7]. In this letter, the Mach-Zehnder modulators fabricated by gallium-diffusion in a y-cut $\mathrm{LiNbO}_{3}$ substrate are presented. The halfwave voltage and the extinction ratio are measured. By calculating the overlap integral of the optical and modulating electric fields, the EO coefficient $r_{33}$ in gallium-diffused waveguide is found in good agreement with that of the bulk $\mathrm{LiNbO}_{3}$, which indicates no significant degradation in $r_{33}$ is induced by the in-diffused gallium atoms.

\section{DEVICE FABRICATION}

To utilize the largest EO coefficient $r_{33}$, a Mach-Zehnder modulator is fabricated in a y-cut $x$-propagating $\mathrm{LiNbO}_{3}$ substrate with a pair of symmetric coplanar strip electrodes. The device configuration is shown in Fig. 1. For single-mode operation, the waveguide width is chosen as $4 \mu \mathrm{m}$. The length of the two parallel waveguide arms is $1 \mathrm{~cm}$ and the full-branch angle is $1^{\circ}$. For simplicity, a pair of electrodes is used instead of a set of three push-pull electrodes. The length and gap width of the electrodes are chosen as $0.4 \mathrm{~cm}$ and $10 \mu \mathrm{m}$, respectively. The fabrication process is described as follows. First, the device pattern is transferred to the $\mathrm{LiNbO}_{3}$ substrate by the standard photolithography process. Then, a $\mathrm{Ga}_{2} \mathrm{O}_{3}$ thin film is deposited by radio-frequency sputtering as the diffusion source. The diffusion process is performed in a furnace at $900^{\circ} \mathrm{C}$ for $1.5 \mathrm{~h}$. Then, the end faces are polished by diamond films. Finally, a pair of aluminum electrodes is deposited by thermal evaporation.

\section{MEASUREMENT AND Results}

For optical characterization, a He-Ne laser of wavelength $632.8 \mathrm{~nm}$ is coupled through a polarizer to the end face of the Mach-Zehnder interferometer. A symmetric triangular voltage 


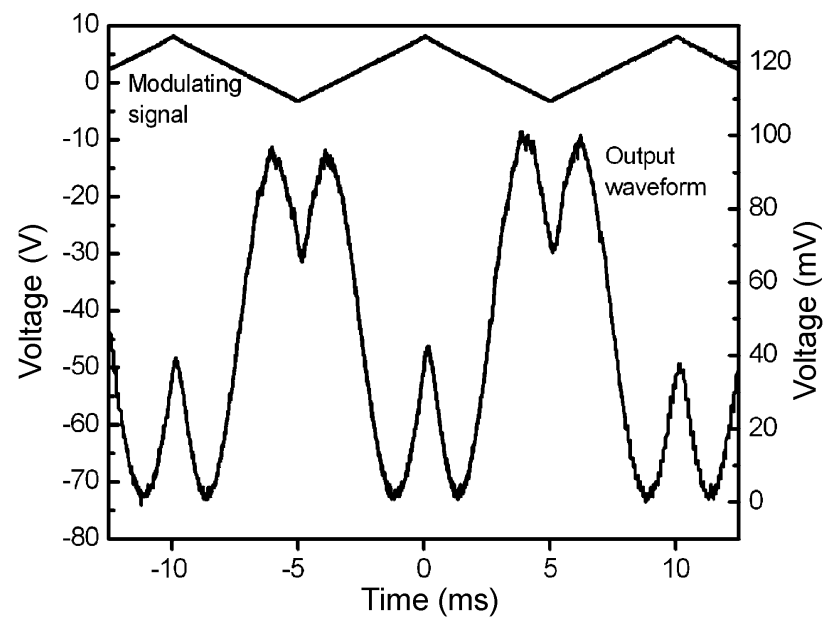

(a)

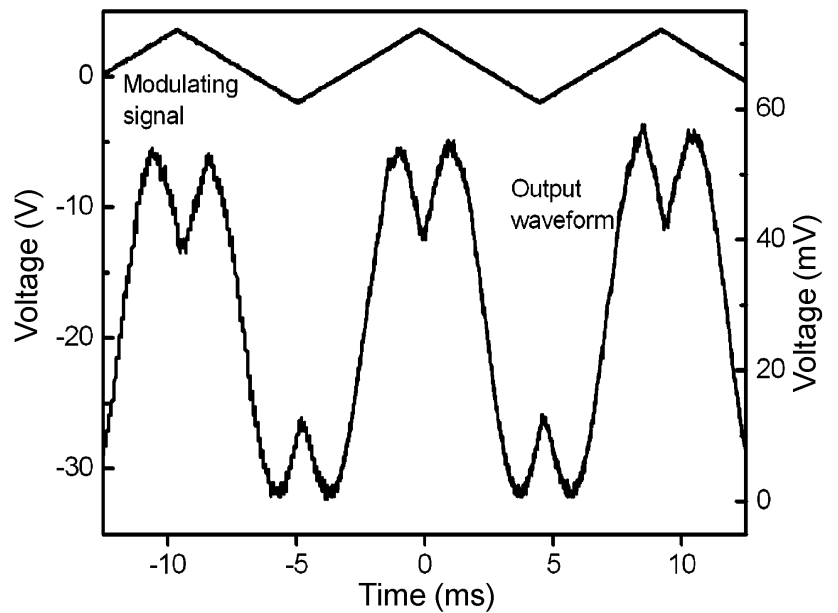

(b)

Fig. 2. Optical response of the Mach-Zehnder modulator driven by a symmetric triangular voltage signal with a pair of (a) 4- and (b) 8-mm-long electrodes.

signal is then applied to the electrodes. The applied voltage waveform and modulated output signals are displayed on an oscilloscope as shown in Fig. 2 . The halfwave voltage $\left(V_{\pi}\right)$ and the extinction ratio are measured to be $6.2 \mathrm{~V}$ and $20.8 \mathrm{~dB}$, respectively. Another modulator with a pair of $0.8-\mathrm{cm}$-long electrodes is also fabricated for comparison. The halfwave voltage and the extinction ratio are measured to be $3.2 \mathrm{~V}$ and $21.4 \mathrm{~dB}$, respectively. Note that, as the gallium-diffused waveguides have been shown to support only extraordinary waves [7], the output power of ordinary waves can hardly be detected.

For a Mach-Zehnder modulator, the halfwave voltage is written as [8]

$$
V_{\pi}=\frac{\lambda G}{n^{3} r_{33} \Gamma L}
$$

where $\lambda$ is the operating wavelength, $n$ is the refractive index of the waveguide, $L$ is the length of the electrodes, and $\Gamma$ is the overlap integral between the optical and modulating electric fields as defined by the following equation [9]:

$$
\Gamma=\frac{G}{V} \frac{\iint E_{\mathrm{op}}^{2}(x, y) E_{\mathrm{el}}(x, y) d x d y}{\iint E_{\mathrm{op}}^{2}(x, y) d x d y}
$$

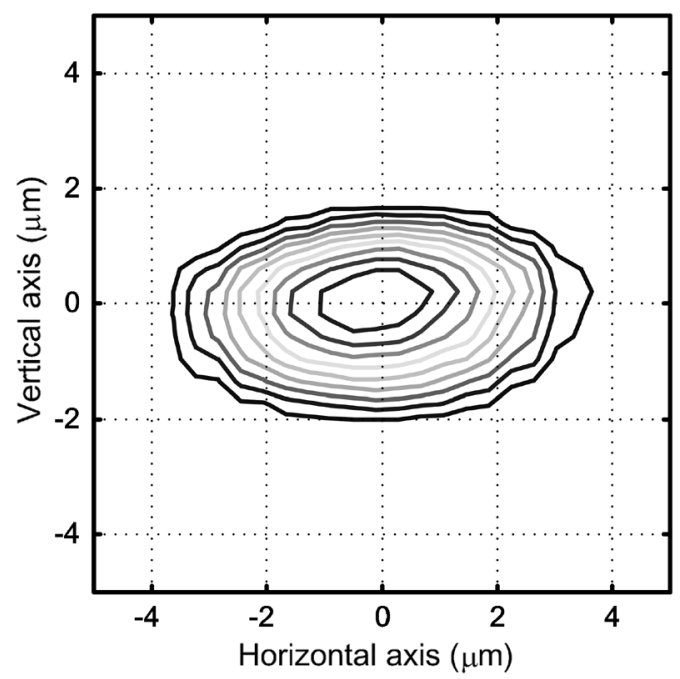

Fig. 3. Optical field intensity contours of the modulator.

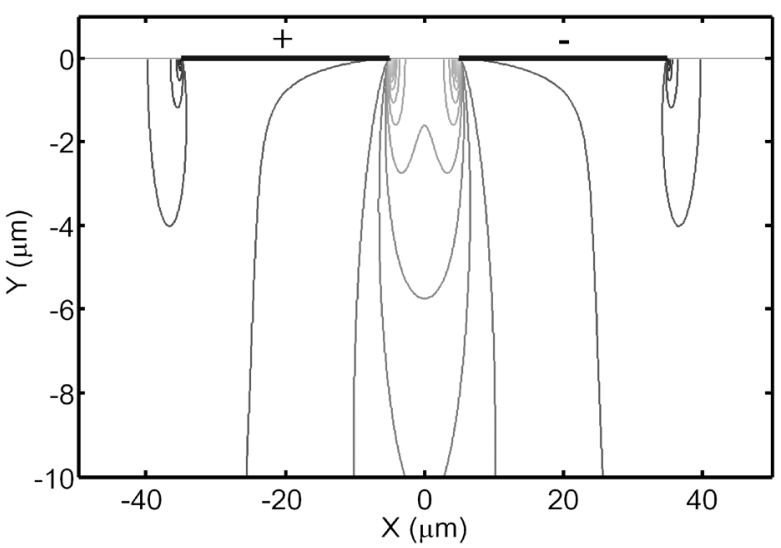

Fig. 4. Simulated electric field contour profiles of a modulator with an applied voltage.

where $G$ is the gap between the electrodes, $V$ is the applied voltage, $E_{\mathrm{op}}(x, y)$ is the optical field, and $E_{\mathrm{el}}(x, y)$ is the electric field. Fig. 3 shows the measured optical field intensity contours of the modulator. The simulated electric field is shown in Fig. 4. In this case, $\Gamma$ and $n$ are found to be 0.7465 and 2.206, respectively. As a result, the calculated values of $r_{33}$ are 30.84 and $31.84 \mathrm{pm} / \mathrm{V}$ for the $0.8-$ and $0.4-\mathrm{cm}-$ long electrodes, respectively. Thus, good agreement between the estimated and bulk values of $r_{33}$ is obtained, indicating that no significant degradation in $r_{33}$ is induced by the in-diffused gallium atoms in $\mathrm{LiNbO}_{3}$ substrate. In addition, the total loss of the device, characterized by comparing the transmitted power of a modulator and a straight-channel waveguide on the same substrate, is about $3.4 \mathrm{~dB}$. To achieve a lower driving voltage, push-pull electrodes and ridge waveguide structure [10] have been demonstrated to be an effective solution. Moreover, the device length can be shortened by introducing a simplified coherently coupled bending structure [11] to the Y-branch region of the Mach-Zehnder modulator.

It is known that the value of $r_{33}$ in the proton-exchanged waveguide is severely degraded to one tenth of its bulk value [5]. Even with an additional postexchanged annealing process, 
TABLE I

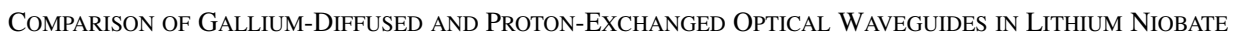

\begin{tabular}{cccc}
\hline \hline Parameter & Gallium-diffusion [7] & Proton-exchange & Annealed proton-exchange \\
\hline Index distribution & Graded & Step & Graded \\
Maximum index change & $10^{-2} \sim 10^{-3}$ & $10^{-1}$ & $10^{-2}$ \\
& & $0.5(\mathrm{x}-\mathrm{cut})[12]$ & $0.15(\mathrm{x}$-cut $)[15]$ \\
Propagation loss (dB/cm) & $0.2 \sim 0.9$ (y-cut) & $3(\mathrm{y}$-cut) [13] & 0.15 (z-cut) [14] \\
Annealing Process & No & No & Yes \\
$r_{33}$ degradation & No & $90 \%$ degradation $[5]$ & $25 \%$ degradation $[6]$ \\
\hline \hline
\end{tabular}

only $75 \%$ restoration of $r_{33}$ can be obtained [6]. A comparison of gallium-diffused, proton-exchanged, and annealed protonexchanged optical waveguides in lithium niobate is listed in Table I. It can be seen that gallium-diffused waveguides are good alternatives for making EO devices for practical application.

\section{CONCLUSION}

The EO coefficient $r_{33}$ of the gallium-diffused waveguide on $\mathrm{LiNbO}_{3}$ substrate is investigated. A Mach-Zehnder optical modulator is fabricated by the gallium-diffused waveguide in a y-cut, x-propagating $\mathrm{LiNbO}_{3}$ substrate with a pair of symmetric coplanar strip electrodes. The halfwave voltages are measured to be 6.2 and $3.2 \mathrm{~V}$ for $0.4-$ and $0.8-\mathrm{cm}-$ long electrodes, respectively. Good agreement between the estimated and theoretical values of $r_{33}$ indicates that no significant degradation in $\mathrm{EO}$ effect is induced by the gallium atoms diffused into $\mathrm{LiNbO}_{3}$ substrate. Moreover, with the low propagation loss, singly guided polarization, and high EO coefficient, gallium-diffused waveguides are demonstrated to be suitable for EO-device application.

\section{REFERENCES}

[1] N. Kuzuta and K. Takakura, "Polarization insensitive optical devices with power splitting and switching functions," Electron. Lett., vol. 27, no. 2, pp. 157-158, Jan. 1991.

[2] A. Kaplan and S. Ruschin, "Layout for polarization insensitive modulation in $\mathrm{LiNbO}_{3}$ waveguides," IEEE J. Sel. Topics Quantum Electron., vol. 6, no. 1, pp. 83-87, Jan./Feb. 2000.

[3] J. Saulnier, F. Huet, L. Rivere, M. Carre, and G. Gaumont, "Interferometric-type polarization splitter on Z-propagating $\mathrm{LiNbO}_{3}$ : Ti," Electron. Lett., vol. 26, no. 23, pp. 1940-1941, Nov. 1990.
[4] C.-C. Chen, H. Porte, A. Carenco, J.-P. Goedgebuer, and V. Armbruster, "Phase correction by laser ablation of a polarization independent $\mathrm{LiNbO}_{3}$ Mach-Zehnder modulator," IEEE Photon. Technol. Lett., vol. 9, no. 10, pp. 1361-1363, Oct. 1997.

[5] M. Minakata, K. Kumagai, and S. Kawakami, "Lattice constant changes and electrooptical effects in proton exchanged $\mathrm{LiNbO}_{3}$ optical waveguides," Appl. Phys. Lett., vol. 49, no. 16, pp. 992-994, Oct. 20, 1986.

[6] I. Savatinova, S. Tonchev, R. Todorov, M. N. Armenise, V. M. N. Passaro, and C. C. Ziling, "Electro-optic effect in proton exchanged $\mathrm{LiNbO}_{3}$ and $\mathrm{LiTaO}_{3}$ waveguides," J. Lightw. Technol., vol. 14, no. 3, pp. 403-409, Mar. 1996.

[7] W. H. Huang and W. S. Wang, "Gallium in-diffusion for the fabrication of lithium niobate optical waveguides," IEEE Photon. Technol. Lett., vol. 19, no. 20, pp. 1679-1681, Oct. 15, 2007.

[8] R. G. Hunsperger, Integrated Optics: Theory and Technology, 5th ed. New York: Springer, 2002, ch. 9.

[9] C. M. Kim and R. V. Ramaswamy, "Overlap integral factors in integrated optic modulators and switches," J. Lightw. Technol., vol. 7, no. 7, pp. 1063-1070, Jul. 1989.

[10] S. J. Chang, C. L. Tsai, Y. B. Lin, J. F. Liu, and W. S. Wang, "Improved electro-optic modulator with ridge structure in $\mathrm{x}$-cut $\mathrm{LiNbO}_{3}$," J. Lightw. Technol., vol. 17, no. 5, pp. 843-847, May 1999.

[11] J. J. Su and W. S. Wang, "Novel coherently coupled multisectional bending optical waveguide," IEEE Photon. Technol. Lett., vol. 14, no. 8, pp. 1112-1114, Aug. 2002.

[12] J. L. Jackel, C. E. Rice, and J. J. Veselka, "Proton exchange for highindex waveguides in $\mathrm{LiNbO}_{3}$," Appl. Phys. Lett., vol. 41, no. 7, pp. 607-608, Oct. 1982.

[13] M. Goodwin and C. Stewart, "Proton-exchanged optical waveguides in Y-cut lithium niobate," Eletron. Lett., vol. 19, no. 6, pp. 223-224, Mar. 1983.

[14] N. Gato and G. L. Yip, "Characterization of proton-exchange and annealed $\mathrm{LiNbO}_{3}$ waveguides with pyrophosphoric acid," Appl. Opt., vol. 28, no. 1, pp. 60-65, Jan. 1989.

[15] P. G. Suchoski, T. K. Findakly, and F. J. Leonberger, "Stable low-loss proton-exchanged $\mathrm{LiNbO}_{3}$ waveguide devices with no electro-optic degradation," Opt. Lett., vol. 13, no. 11, pp. 1050-1052, Nov. 1988. 